\title{
Visual perspective in autobiographical memories of self-incongruent episodes
}

\author{
Thomas Lukaschewski ${ }^{1}$, Gerd Waldhauser ${ }^{1}$, Roy Dings ${ }^{2}$, Rebekka Heinen ${ }^{1}$, Albert Newen ${ }^{2}$, \\ Nikolai Axmacher ${ }^{1}$ \\ ${ }^{1}$ Department of Neuropsychology, Institute of Cognitive Neuroscience, Faculty of Psychology, Ruhr University Bochum, Bochum, Germany \\ ${ }^{2}$ Institute of Philosophy II, Ruhr Universität Bochum, Bochum, Germany
}

\begin{abstract}
Rationale: It is widely assumed that autobiographical memories rely on an integration of episodic memories with the self-model. We hypothesize that this integration process is influenced by the affective valence and the specific emotions that are elicited by an experience. More specifically, negative experiences that are incongruent with the self-model and that elicit self-conscious emotions such as shame or guilt may be more difficult to integrate. This self-conflict could not only affect the content of autobiographical memories, but also their phenomenology: Apart from their affective valence, autobiographical memories differ in their visual perspective, because they can either be experienced from a first or a third person perspective (field vs. observer perspective), as well as in their perceived centrality for one's life story. Understanding whether and how activation of, and incongruence with, the self-model influences the phenomenology of autobiographical memories is thus an important step towards a mechanistic model of self-memory integration.
\end{abstract}

Objective: We aimed to investigate whether self-incongruent autobiographical memories that elicit feelings of shame or guilt differ in their phenomenological characteristics from other negative or neutral memories. We focus on the visual perspective of these memories and aim to elucidate the factors that contribute to possible changes in visual perspective from an initial first to the third person perspective.

Methods: A group of $n=65$ adult participants (age range: 18-35) completed a daily online diary survey over 11 weeks. On each day, participants were asked to report an episode from one of four different categories (shame, guilt, negative, neutral). Participants rated all memories in terms of their degree of shame, guilt, negative affect, visual perspective, and expected centrality. Differences between phenomenological characteristics of the four types of episodes were assessed via ANOVAs, and the relationships between these characteristics were analyzed via hierarchical multilevel models.

Results: Autobiographical memories that elicit feelings of shame, but not those inducing guilt, showed higher degrees of $3^{\text {rd }}$ person visual perspective as compared to negative or neutral experiences. However, shameful episodes also elicited the most pronounced negative affect, and correlation analyses revealed that affective valence, expected centrality and shame were all related to visual perspective. A multilevel analysis in which several predictors of visual perspective were considered conjointly helped disentangle these effects: It showed only a highly significant effect of shame and a marginally significant effect of expected centrality. Affective valence did not have a significant influence on visual perspective in this model, suggesting that its effect on visual perspective is mainly mediated by shame.

Conclusion: We found that incongruence with the self-model affects the phenomenological characteristics of autobiographical memories and show that this effect depends on feelings of shame. Our results provide a better understanding of the factors that influence the visual perspective of autobiographical memories. More generally, they contribute to a mechanistic understanding of self-memory integration in the service of autobiographical memory. Future research should address whether and how changes in visual perspective can be manipulated experimentally, how they develop over time, and whether they contribute to psychopathology such as memories for traumatic events. 


\section{Introduction}

Self-integration has been defined as a prerequisite for an experience to become part of a person's identity (McAdams \& Pals, 2006). This can only happen if those experiences are included in the self-defining life narrative, which requires a process of self-memory integration (McAdams, 1996). In other words, self-memory integration aligns experiences with personal goals and current knowledge and allows for the encoding of experiences into autobiographical long-term memory (Conway \& Pleydell-Pearce, 2000). A mechanistic understanding of this process requires examining those cases in which integration is not effortlessly possible. This is the case for so-called self-discrepant or self-incongruent experiences in which memory contents conflict with central aspects of the self (Singer \& Blagov, 2004).

According to Tory Higgins (1987), different types of self-discrepancies may result from conflicts between a particular autobiographical memory (AM) and different aspects of the self: Memories may conflict either with a person's self-ideal (ideal-conflict) or with their assumed social expectations (ought-conflict). More recent research supports a similar distinction concerning the experience of so-called self-conscious emotions (Leary, 2007). In contrast to the basic emotions of fear, joy, and anger, the self-conscious emotions shame and guilt are often categorized as secondary or complex because they develop at a later point in life, depending on a child's cognitive and emotional development (Zinck \& Newen 2008; Tangney, 2015). Together with pride and embarrassment, these emotions are termed "self-conscious" because of the widely accepted idea that self-reflection is needed to evoke or strengthen them (Kilborne, 1997; Robins \& Schriber, 2009). Research by Tangney and others has resulted in the most commonly applied distinction between shame and guilt, according to which shame is inherently self-related while guilt is associated with a specific behavioral action (Tangney et al., 1992; 1996). This proposal can be linked to Higgins' view of the two types of self-conflict; because ideal-conflicts may be more inherently self-related, while ought-conflicts may be triggered by specific actions. It should be noted, however, that alternative accounts on the difference between shame and guilt have been proposed (e.g., Teroni \& Deonna, 2008).

If experiences that elicit feelings of shame or guilt conflict with the self, they may be more difficult to integrate and result in altered phenomenological characteristics of these memories. Indeed, autobiographical memories can be both characterized by their specific content (the episode that I remember) and their phenomenological characteristics (how I remember this episode). Self-memory integration may affect the content of an autobiographical memory e.g., modifying it such that it appears more congruent with the self-model (Conway \& PleydellPearce, 2000; Conway, 2005). However, if this results in blatantly "false memories", obvious problems may arise. A more subtle and sustainable strategy may consist in a cognitive distancing from these episodes, which is consistent with actual episodes at the level of the content but alters their phenomenological appearance. Since shame has been associated with various psychopathologies, one may further assume that these phenomenological changes are particularly pronounced for memories of shameful episodes (Gilbert, 2000; Mills, 2015).

More specifically, we hypothesized that self-incongruence may induce a shift in the visual perspective of autobiographical memories from a first to a third person view. Visual perspective can be considered a defining aspect of the phenomenal self (e.g., Metzinger, 2007) together with the sense of agency, the sense of ownership, etc. (Synofzik et al. 2008). While most everyday experiences are encoded in a first-person perspective (1PP), the visual perspective of memories may subsequently change to a third-person perspective (3PP). This change can occur automatically as a function of time (Nigro \& Neisser, 1983) or result from deliberate efforts (Robinson \& Swanson, 1993; St. Jacques, Szpunar \& Schacter, 2017). Importantly, already the 
seminar study by Nigro and Neisser (1983) showed that higher levels of self-awareness during an experience increase its later recall from 3PP, suggesting that 3PP reflects an explicit representation of the self-model into a remembered episode.

Why may episodic memories be recalled from different visual perspectives? The function of 3PP in episodic memory recall has been repeatedly investigated (for review, see Sutin \& Robins, 2008; Libby \& Eibach, 2011). One study investigated effects of self-evaluation on visual perspective and reported stronger 3PP for episodes involving either positive or negative self-evaluations (i.e., pride or shame) than for those that involve evaluations of others (D'Argembeau \& van der Linden, 2008). Other studies suggest that this effect may be more pronounced for episodes that involved a discrepancy between perceived and desired self-model because healthy participants particularly remembered those episodes from a 3PP that contained a behavior or a trait which they hoped to change in the future (Libby \& Eibach, 2002; Libby, Eibach \& Gilovich, 2005). Further studies suggest that remembering an event from a 3PP serves to reduce negative affect: This was shown both for traumatic events that were spontaneously recalled from a 3PP (Berntsen, Willert \& Rubin, 2003; McIsaac \& Eich, 2004) and for non-traumatic experiences that were deliberately recalled from a 3PP (Robinson \& Swanson, 1993; McIsaac \& Eich, 2002; Berntsen \& Rubin, 2006a; St. Jacques et al., 2017). Finally, recall from a 3PP changes the causal attribution of actions by increasing dispositional attributions, potentially because in 3PP memories the self is perceived as part of the remembered episode (Frank \& Gilovich, 1989).

Together, these studies suggest that retrieving memories from a 3PP either serves an emotional distancing function for memories that conflict with the self-model (Sanitioso, 2008; Holmes \& Mathews, 2010; St. Jacques et al., 2017) or reflects cognitive processes related to self-reflection more generally (Libby \& Eibach, 2011). With regard to shame and guilt, self-incongruent memories have been proposed to elicit changes in visual perspective (Libby \& Eibach, 2002; Sutin \& Robins, 2008). Several studies showed that highly emotional and stressful situations that involve a focus on the self were more likely to be recalled from 3PP (Nigro \& Neisser, 1983; D’Argembeau \& Van der Linden, 2008; Sutin \& Robins, 2008). These effects may be adaptive in that they allow distancing from self-incongruent experiences (Wilson, Ross, \& Wilson, 2010). However, whether they are particularly related to feelings of shame, guilt, or both has remained unclear thus far.

Another phenomenological measure that putatively reflects distancing from self-incongruent experiences is an overall reduction in negative emotions. Autobiographical memories differ in their affective valence. While episodes and memories that are accompanied by negative selfconscious emotions are most likely among the most distressing experiences of our life, it is not clear whether the underlying emotions elicit more pronounced negative valence than other negative episodes such as those that elicit anger, fear, or sadness (Hepach et al., 2011). In addition, whether and how negative valence affects visual perspective is still unclear.

Finally, autobiographical memories differ in their perceived centrality for one's life. Berntsen and Rubin (2006) developed the centrality of events scale (CES) to measure the degree to which memories become an anchor point for personal identity and for meaning in a person's life. The CES has been used repeatedly to relate the relevance of various positive and negative experiences to people's life-narratives (Zaragoza Scherman et al., 2014; Gehrt et al., 2018). While it is likely that episodes which are experienced as more central elicit more pronounced self-reflection, it is still an open question whether event centrality elicits shifts in visual perspective. 
In the current study, we collected real-world autobiographical memories and investigated the phenomenological characteristics of self-incongruent memories in contrast to negative and neutral memories which are not self-incongruent. We particularly focused on visual perspective, but additionally considered other variables that may mediate or moderate the influence of self-incongruence on visual perspective. First, we hypothesized a higher degree of $3 \mathrm{PP}$ in self-incongruent than self-congruent memories. Second, we predicted that shameinducing autobiographical memories may elicit particularly high degrees of 3PP because the need for distancing may be more pronounced for episodes that conflict with central aspects of the self. Third, we tested whether higher degrees of expected event centrality and higher negative affect ratings increase $3 \mathrm{PP}$.

\section{Methods}

\section{Participants}

Participants were recruited via notices on bulletin boards on the campus of Ruhr University Bochum and through an external regional online platform (Stellenwerk). Additional participants were recruited by distributing flyers and via social media. To ensure that potential participants met all requirements, a brief telephone screening was carried out.

$\mathrm{N}=65$ adult participants agreed to participate in exchange for a compensation of $100 €$. Participants who repeatedly failed to make entries in the diary were excluded from the study ( $\mathrm{n}=15$ participants).

Exclusion criteria were current or previous neurological diseases, cardiovascular diseases, age $<18$ or $>35$, and lack of German knowledge. Due to the study design, participants had to own a smartphone. The study was approved by the ethical committee of the Faculty of Psychology of Ruhr University Bochum, and all participants provided written informed consent. An individual code was made available to all participants, allowing them to collect data pseudonymously.

Due to complications related to COVID-19 restrictions, the acquisition phase was subsequently extended by two weeks, amounting to 11 weeks in total. Participants were free to decide whether they wanted to extend their participation. Those who agreed to the extended acquisition period were rewarded with an additional 10 Euros.

\section{Experimental Procedure}

One week before the start of the study, all participants were invited to a two-hour introductory session. The researchers answered questions about the study, and all documents were filled out and signed by the participants. Participants then completed a baseline survey including demographic items and were familiarized with the use of the online diary. A description was included on how shame and guilt experiences can typically be characterized, and participants were asked to report some example episodes from their past. In addition, they were asked to justify why they would place the experience in one of the relevant categories. However, we explicitly avoided giving a precise specification about which categorization is correct. This should ensure that the widest possible variety of self-incongruent experiences was collected. 
For the next nine or eleven weeks (see above), each participant received a daily reminder at 7 pm with a link to an online diary tool based on Google forms. Google forms is a survey administration software included as part of the free, web-based Google Docs Editors suite. It enables the creation of online forms with which standardized questionnaires can be brought into a digital form and with which both compliance with the time frame and the assignment to the previously generated test person codes can be tracked. Participants had time until $2 \mathrm{am}$ the following day to provide reports. If they failed to submit reports within this time window, they could only add missing items when providing specific justification. After the diary collection was finished, a three-part experimental manipulation followed, but this could not be carried out as planned due to COVID-19 restrictions and is not reported here.

To ensure that a sufficient number of self-incongruent experiences were collected, the participants were instructed to select a shameful or guilt-related experience of the current day after receiving their daily reminder email and to select the associated categories (shame or guilt). Only if no episode of one of these two categories could be reported, a different negative emotional episode was to be chosen (i.e., one that did not elicit feelings of shame or guilt, but e.g., anger or sadness). In this case, the category "negative (neither shame nor guilt)" was to be selected. If no negative emotional experience could be reported, the participants was asked to choose a unique neutral experience from the past day and report the category "neutral".

After selecting the episode category, participants were asked to write down their selected episode of the current day in detail and to assign a clear keyword to their entry. This measure was only included for the later experimental manipulation and is thus not considered further.

As described by (Miceli \& Castelfranchi, 2018), self-incongruent experiences can evoke both shame and guilt at the same time, and some of the "generally negative" or neutral episodes may elicit some amounts of shame and/or guilt as well. For this reason, the subjects were instructed to rate levels of shame and guilt for every experience, regardless of the chosen episode category. A 5 -point Likert scale $(1=$ not at all to $5=$ very strongly $)$ was used for this purpose.

The negative affect of each diary entry was determined using a short version of the Positive and Negative Affect Schedule (PANAS-SF) (Thompson, 2007). The five negative items are averaged and formed the "negative affect" variable.

Studies by St. Jacques et. al. $(2017 ; 2018)$ have shown that the visual perspective often cannot be dichotomized to a "pure" first or third-person perspective and that a continuous rating scale is thus more appropriate. A 5-point Likert scale was used to assess the visual perspective of the daily experiences $(1=$ completely first person; $5=$ completely third-person perspective).

Finally, participants completed a revised version of the Centrality of Events Scale (CES) (Berntsen \& Rubin, 2006b). In its original form, this questionnaire is used to assess the relevance of autobiographical memory concerning other experiences and the participant's selfidentity (i.e., self-model). Since the participants wrote down new experiences, the individual questions were reworded in such a way that the test persons should assess the expected future relevance of the respective event.

Thus, each recorded episode was associated with six ratings: (1) Categorical episode type (shame, guilt, negative, or neutral), (2) 5-point measure of shame, (3) 5-point measure of guilt, (4) valence (negative affect), (5) visual perspective, and (6) expected centrality. 


\section{Data Analysis}

In order to assess differences in visual perspective, negative affect, shame, guilt, and expected centrality between the 4 episode categories, we applied 5 separate repeated measures ANOVAs on all episodes of a given category (averaged per participant). In order to correct for possible lack of sphericity, we applied Greenhouse-Geisser correction. In case of significant effects, post-hoc t-tests were conducted using Bonferroni correction for multiple comparisons. This analysis was conducted using SPSS 28 (IBM SPSS Statistics for Mac, Version 28.0).

Next, we assessed which phenomenological properties of the different episodes predicted the visual perspective (i.e., amount of 3PP) of a given episode. Since this analysis had to be conducted at the level of individual episodes, we applied hierarchical multi-level analyses with "visual perspective" as dependent variable and episode-specific ratings of valence, centrality, shame, guilt as independent variables. Since the number of episodes per category as well as the overall ratings differed between participants, we included "participant" as a nested variable, within which "episode type" (shame, guilt, negative, neutral) was a second nested variable. These analyses were conducted in R Studio (R Core Team, 2019) using the 1me4, lmerTest, emmeans and sjPlot packages.

\section{Results}

In total, we collected 3,245 autobiographical memories from 50 participants (shame: $n=435$, range: 0 -22; guilt: $n=520,0-26$; negative: $n=796,2-45$; neutral: $n=1,494,2-63$ ). First, we compared affect ratings between the different types of episodes. As expected, negative affect ratings differed between categories (Figure 1A; $\mathrm{F}(2.56,117.71)=129.91, \mathrm{p}<.001$ ). Bonferronicorrected post-hoc t-tests indicated that negative affect ratings were significantly higher for shame episodes $(M: 2.39 \pm S D: 0.52)$ compared with episodes that elicited guilt $(M: 2.14 \pm$ $S D: 0.59 ; \mathrm{p}=.004)$ or other negative feelings $(M: 2.08 \pm S D: 0.44 ; \mathrm{p}<.001)$ or that were neutral $(M: 1.24 \pm S D: 0.23 ; \mathrm{p}<.001)$. Both guilt-inducing episodes and negative episodes elicited higher negative affect ratings than neutral episodes (both $\mathrm{p}<.001$ ). Negative affect ratings did not differ between guilt-inducing episodes and other negative episodes. 
A)

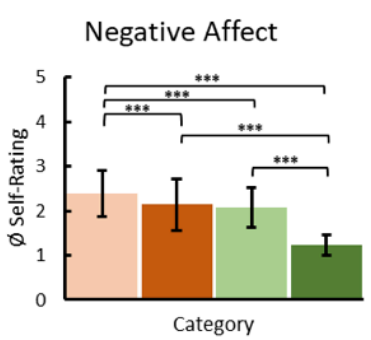

D)

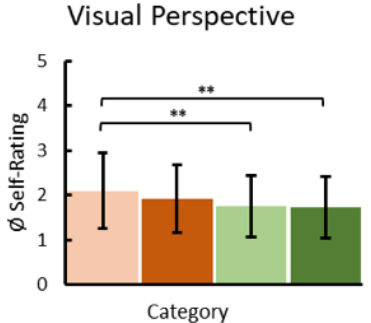

B)

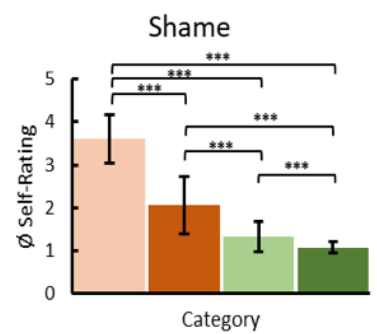

E)

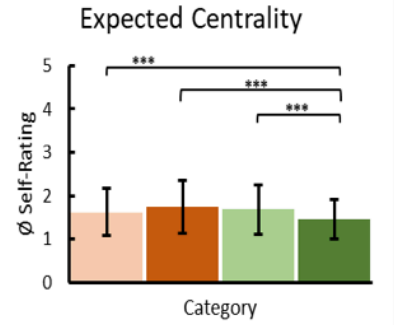

C)

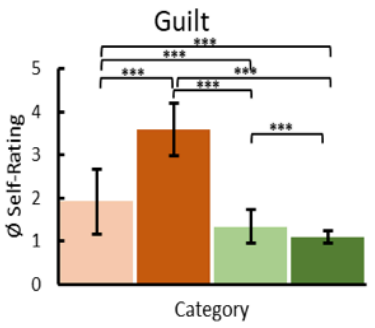

Figure 1: Phenomenological characteristics of autobiographical memories of different types of episodes (shame, guilt, negative, neutral). A) Negative affect; B) Shame ratings; C) guilt ratings; D) expected centrality; E) visual perspective. Bonferroni-corrected post-hoc t-tests $(* * * p<.001 ; * * p<.01)$.

We also compared ratings of shame and guilt between the different episodes. As expected, ratings of "shame" differed between episodes; $\mathrm{F}(2.17,99.63)=341.018, \mathrm{p}<.001$. Bonferronicorrected post-hoc t-tests indicated that shame ratings were significantly higher for shame

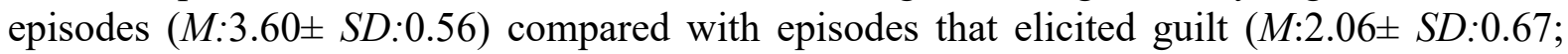
$\mathrm{p}<.001)$ or other negative feelings $(M: 1.33 \pm S D: 0.36 ; \mathrm{p}<.001)$ or that were neutral $(M: 1.08 \pm$ $S D: 0.13 ; \mathrm{p}<.001)$. Shame ratings for guilt episodes were significantly higher compared with episodes that elicited negative feelings $(\mathrm{p}<.001)$. Both guilt-inducing episodes and negative episodes elicited higher shame ratings than neutral episodes (both $\mathrm{p}<.001$ ) (Figure 1B).

Ratings of "guilt" also differed between episodes; $F(2.08,95.49)=276.833, \mathrm{p}<.001$. Bonferronicorrected post-hoc t-tests indicated that guilt ratings were significantly higher for guilt episodes $(M: 3.59 \pm S D: 0.62)$ compared with episodes that elicited shame $(M: 1.92 \pm S D: 0.76 ; \mathrm{p}<.001)$ or other negative feelings $(M: 1.33 \pm S D: 0.39 ; \mathrm{p}<.001)$ or that were neutral $(M: 1.09 \pm S D: 0.14$; $\mathrm{p}<.001)$. Guilt ratings for shame episodes were significantly higher compared with episodes that elicited negative feelings $(\mathrm{p}<.001)$. Both shame-inducing episodes and negative episodes elicited higher guilt ratings than neutral episodes (both $\mathrm{p}<.001$ ) (Figure $1 \mathrm{C}$ ).

Interestingly, we also found that visual perspective differed significantly between episode categories (Figure 1D; $\mathrm{F}(2.43,111.97)=6.965, \mathrm{p}<.001)$. Post-hoc t-tests revealed significantly more pronounced 3PP ratings for shame episodes (M:2.10 $\pm S D: 0.84)$ compared to either negative episodes $(M: 1.76 \pm S D: 0.69 ; \mathrm{p}=.002)$ or neutral episodes $(M: 1.73 \pm S D: 0.69 ; \mathrm{p}=.004)$. None of the other pairwise comparisons of visual perspective ratings between episode categories showed significant differences. 
We also found that ratings of expected centrality differed between episode categories (Figure $1 \mathrm{E} ; \mathrm{F}(2.71,124.61)=13.673, \mathrm{p}<.001)$. Post-hoc t-tests showed that expected centrality ratings were significantly higher for shame episodes $(M: 1.62 \pm S D: 0.55)$, guilt episodes $(M: 1.74 \pm$ $S D: 0.60)$ and negative episodes $(M: 1.68 \pm S D: 0.57)$ compared to neutral episodes $(M: 1.45 \pm$ $S D: 0.45$; all $\mathrm{p}<.001)$. None of the other pairwise comparisons of expected centrality ratings between episode categories showed significant differences.

Our analyses presented so far showed that memories of shameful episodes relied significantly more on 3PP than memories of negative or neutral events, while guilt-inducing episodes ranged in-between shameful and negative/neutral memories (and differed from neither). Since we also found differences in negative valence between shameful episodes and all other episodes, and in centrality between shameful episodes and neutral episodes, the question arises whether negative affect and centrality account for the effect of shameful episodes on visual perspective. In order to address these questions, we tested whether the negative affect and the centrality of individual episodes predicted their visual perspective, and if so, whether the effect of shame on visual perspective remains after considering possible effects of negative affect and centrality.

\section{Multi-level analyses}

We thus conducted hierarchical multi-level analyses in which the visual perspective of each episode was used as dependent variable. The factor "subject" was modeled as a nested random intercept variable (because different participants may differ in their propensity to remember episodes from a 3PP, and because the number of episodes of the individual categories differed between participants). Further, we added categories (shame, guilt, negative, neutral) as random slopes which allows for a different slope for the visual perspective ratings depending on the episode-type.

We applied four parallel multi-level models. In addition to the random effect, each model contained a fixed predictor to describe the relationships between the visual perspective and the individual phenomenological variables (shame, guilt, expected centrality, negative affect) (see table 1). The results show significant correlations between expected centrality, negative affect, and shame, but not guilt, with the dependent variable (visual perspective). There is no significant association between guilt ratings and visual perspective within our multilevel model.

\section{Table 1: Multilevel correlations}

\begin{tabular}{lllll} 
& \multicolumn{4}{l}{ Correlations } \\
\cline { 2 - 5 } & $\begin{array}{l}\text { Negative } \\
\text { Affect }\end{array}$ & $\begin{array}{l}\text { Expected } \\
\text { Centrality }\end{array}$ & Shame & Guilt \\
\cline { 2 - 5 } Visual & $.09 * * *$ & $.07 * *$ & $.12 * * *$ & $<.01$ \\
Perspective & & & &
\end{tabular}

Table1: Notes: $* * p<.01 ; * * * p<.001$ 
In the next step, we applied a hierarchical (multi-level) multiple linear regression analysis in order to conjointly consider the effects of all variables on visual perspective. We again used visual perspective as dependent variable, but in this case, we added all other variables (category, shame, guilt, expected centrality, and negative affect) as fixed effect predictors. We again included "subject" as random intercept and "category" as random slope. This model showed that the visual perspective ratings of autobiographical memories were significantly predicted by expected centrality and, more prominently, by the subjective shame ratings, while none of the other predictors reached significance (see Table 2, Figure 2 A-E)

Table 2: Fixed and random effects

\begin{tabular}{lccc}
\hline & \multicolumn{3}{c}{ Effects on visual perspective ratings } \\
Predictors & Estimates & $C I$ & $p$ \\
\hline (Intercept) & 1.71 & $1.36-2.05$ & $<\mathbf{0 . 0 0 1}$ \\
Episode Category & -0.04 & $-0.11-0.04$ & 0.315 \\
Expected Centrality & 0.06 & $0.00-0.11$ & $\mathbf{0 . 0 4 9}$ \\
Negative Affect & 0.03 & $-0.03-0.09$ & 0.321 \\
Shame rating & 0.10 & $0.05-0.15$ & $<\mathbf{0 . 0 0 1}$ \\
Guilt rating & -0.03 & $-0.07-0.01$ & 0.109 \\
Random Effects & & & \\
$\sigma^{2}$ & 0.82 & & \\
$\tau_{00}$ Participant & 0.56 & & \\
$\tau_{11}$ Participant.Category & 0.03 & & \\
$\varrho_{01}$ Participant & -0.52 & & \\
ICC & 0.36 & & \\
$\mathrm{~N}_{\text {Participant }}$ & 50 & & \\
\hline Observations & 3245 & & \\
Marginal R $^{2}$ / Conditional $\mathrm{R}^{2}$ & $0.018 / 0.372$ & \\
\hline
\end{tabular}

Table 2: Results from multiple linear regression within the multi-level model including all predictors (category, expected centrality, negative affect, shame, and guilt). 
A)

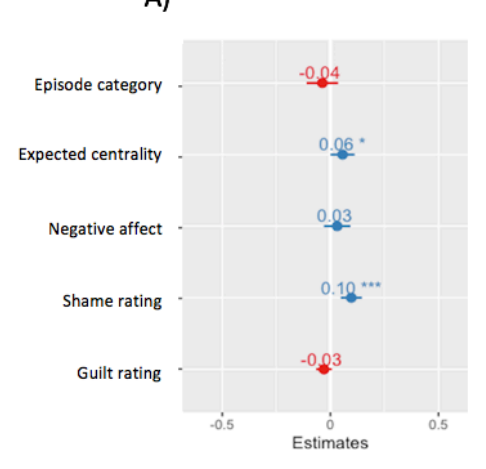

B)

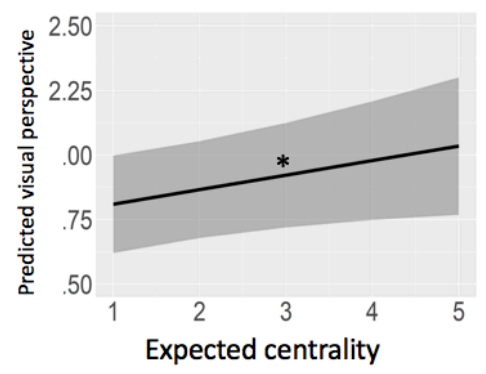

D)

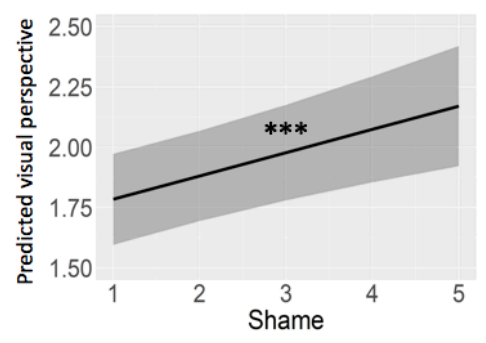

C)

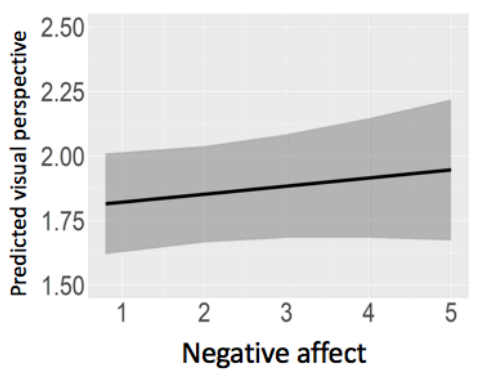

E)

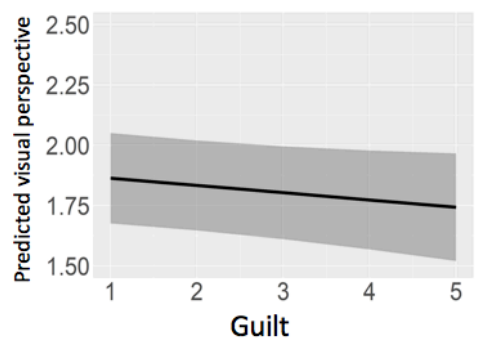

Figure 2A: A visualization of the relationships between the individual predictors (category, negative affect, expected centrality, shame and guilt) on the visual perspective within the multilevel model. Figures B-E: The fitted multilevel model (with Subject as random intercepts and Category as random slope) showing the linear relationship between the individual fixed effects and the outcome variable Visual Perception (shaded area represent standard error). High values on the $y$-axis signify increased $3 P P .(* p<.05 ; * * * p<.001)$.

\section{Discussion}

Here we investigated the influence of self-incongruence on several phenomenological characteristics of autobiographical memories, namely their negative affect, expected centrality, and visual perspective. We also analyzed whether episode-wise ratings of shame and guilt were related to these phenomenological characteristics.

Based on a large data set of real-life autobiographical memories, we could identify phenomenological differences between experiences depending on their emotions (shame, guilt, negative, neutral). Somewhat trivially, experiences in the "shame" category received the highest self-ratings of shame, and experiences in the "guilt" category received the highest selfratings of guilt. More interestingly, we also found that experiences from the "shame" category had higher ratings of guilt, and experiences in the guilt category had higher ratings of shame than experiences in the "negative" category. This shows a substantial overlap between shame and guilt ratings, even though episodes had been selected to be predominantly from one of these categories. Our finding is in line with previous studies showing that a distinction between the self-conscious emotions of shame and guilt produced rather mixed results (Teroni \& Deonna, 2008; Miceli \& Castelfranchi, 2018).

Another interesting finding emerged from the comparison of negative affect ratings between the different episode categories: Shame experiences had significantly higher negative affect ratings when compared to the ratings of the other categories, and therefore constitute the most 
emotionally negative experiences within our data set. These findings can be linked to earlier research in which shameful experiences were classified as particularly emotional and (in more extreme cases than would be expected in our everyday sample) were associated with various psychopathologies (Gilbert, 2000; Schoenleber \& Berenbaum, 2012; Mills, 2015).

Most interestingly, we found significant differences in visual perspective between the different episode categories. Our results show significantly higher 3PP of shame experiences compared to negative and neutral experiences. A similar difference was not apparent when guilt experiences were compared to negative or neutral experiences.

As described in the introduction, visual perspective is influenced by several different factors: Apart from the time since encoding, it increases with self-awareness during an episode (Nigro and Neisser) and may reflect either emotional distancing or self-reflection in general (Sutin \& Robins, 2008). In our study, we found evidence for both interpretations. On the one hand, higher levels of 3PP were predicted by negative emotional valence in general, and with ratings of shame more specifically, supporting the link between 3PP and emotional distancing. On the other hand, levels of 3PP were related to an episode's expected future centrality, which may be more related to enhanced self-reflection. In an overarching model, we found that shame was by far the strongest predictor of 3PP. This finding is particularly important, because it shows that it is not the negative emotionality per se that is fueling changes in visual perspective from $1 \mathrm{PP}$ to $3 \mathrm{PP}$, but that this process is specifically driven by the shamefulness of an experience.

Another significant, though overall less prominent predictor of changes in visual perspective, was the expected centrality of autobiographical memories. Because shame experiences did not show significantly higher values in expected centrality compared to the other emotional categories, this raises the question which other variables influence visual perspective. A possible answer is provided by recent studies by Libby \& Eibach (2011), in which they assume that a visual change in perspective can be triggered solely by a person reflecting upon this experience. Our results on expected centrality would support this interpretation, because experiences with a high expected centrality arguably elicited more pronounced self-reflection, resulting in $3 \mathrm{PP}$.

On a methodological level, our study shows that daily diary entries can be used to distinguish different kinds of self-incongruent experiences and to better understand how visual perspective differs between autobiographical memories. Combined with a multilevel analysis approach, the naturally differing numbers of autobiographical experiences in the different categories and between subjects can also be taken into account, which means that this approach may be a fruitful avenue for further research on the factors that explain the phenomenological characteristics of everyday autobiographical memories.

In the future, follow-up studies should further elucidate the connection between the self and autobiographical memories. For example, categories of self-incongruent experiences can be further refined by creating subcategories. Previous studies have shown that vicarious shame differs greatly from typical shame and that when experiencing guilt, the amount of simultaneously experienced shame depends heavily on the situation (Teroni \& Deonna; 2008 Welten et al., 2012). In addition, it could be shown that different personality types deal very differently with shame experiences (Fontaine et al., 2006; Schalkwijk et al., 2016). Furthermore, the elaboration of additional categories of negative emotional experiences could contribute to a better understanding of the connection between visual perspective and negative affect. Further categories, such as sadness or anger, may be related to the self and could be linked to a kind of gradual self-reference. Finally, the individual diary entries could be 
examined on a semantic level. This could help find semantic relationships between experiences of the same category or of different categories. Semantic connections could then be included in a multilevel model in the form of additional fixed effects.

\section{Conclusions}

The mechanisms underlying self-memory integration are far from being fully understood, and we suggest they can only be unraveled when analyzing the content and phenomenological characteristics of both self-congruent and self-incongruent memories. Our finding that feelings of shame were the strongest predictors of visual perspective suggests that shameful episodes elicit the highest level of self-conflict. Thus, understanding the impact of shame on later memories is relevant for the fundamental question of how memories are integrated with the self-model in the service of autobiographical memory. 


\section{References}

Bates, D., Machler, M., Bolker, B. and Walker, S. (2015) Fitting Linear Mixed-Effects Models Using lme4. Journal of Statistical Software, 67, 1-48. http://dx.doi.org/10.18637/jss.v067.i01

Berntsen, D., \& Rubin, D. C. (2006a). Emotion and vantage point in autobiographical. Cognition and Emotion, 20(8), 1193-1215. https://doi.org/10.1080/02699930500371190

Berntsen, D., \& Rubin, D. C. (2006b). The centrality of event scale: a measure of integrating a trauma into one's identity and its relation to post-traumatic stress disorder symptoms. Behaviour research and therapy, 44(2), 219-231. https://doi.org/10.1016/j.brat.2005.01.009

Berntsen, D., Willert, M., \& Rubin, D. C. (2003). Splintered memories or vivid landmarks? Qualities and organization of traumatic memories with and without PTSD. Applied Cognitive Psychology, 17(6), 675-693. https://doi.org/10.1002/acp.894

Conway, M. A. (2005). Memory and the self. Journal of Memory and Language, 53(4), 594628. https://doi.org/10.1016/j.jml.2005.08.005

Conway, M. A., \& Pleydell-Pearce, C. W. (2000). The construction of autobiographical memories in the self-memory system. Psychological Review, 107(2), 261-288. https://doi.org/10.1037/0033-295X.107.2.261

D'Argembeau, A., \& Van der Linden, M. (2008). Remembering pride and shame: Selfenhancement and the phenomenology of autobiographical memory. Memory, 16(5), 538-547. https://doi.org/10.1080/09658210802010463

Fontaine, J. R. J., Luyten, P., De Boeck, P., Corveleyn, J., Fernandez, M., Herrera, D., Ittzés, A., \& Tomcsányi, T. (2006). Untying the Gordian Knot of Guilt and Shame: The Structure of Guilt and Shame Reactions Based on Situation and Person Variation in 
Belgium, Hungary, and Peru. Journal of Cross-Cultural Psychology, 37(3), 273292. https://doi.org/10.1177/0022022105284493

Frank, M. G., \& Gilovich, T. (1989). Effect of Memory Perspective on Retrospective Causal Attributions. Journal of Personality and Social Psychology, 57(3), 399-403. https://doi.org/10.1037/0022-3514.57.3.399

Gehrt, T. B., Berntsen, D., Hoyle, R. H., \& Rubin, D. C. (2018). Psychological and clinical correlates of the Centrality of Event Scale: A systematic review. Clinical psychology review, 65, 57-80. https://doi.org/10.1016/j.cpr.2018.07.006

Gilbert, P. (2000). The relationship of shame, social anxiety and depression: the role of the evaluation of social rank. Clinical Psychology \& Psychotherapy, 7, 174-189.

Gu, X., \& Tse, C. S. (2016). Narrative perspective shift at retrieval: The psychologicaldistance-mediated-effect on emotional intensity of positive and negative autobiographical memory. Consciousness and Cognition, 45, 159-173. https://doi.org/10.1016/j.concog.2016.09.001

Hepach, R., Kliemann, D., Grüneisen, S., Heekeren, H. R., \& Dziobek, I. (2011). Conceptualizing emotions along the dimensions of valence, arousal, and communicative frequency-Implications for social-cognitive tests and training tools. Frontiers in Psychology, 2, Article 266. https://doi.org/10.3389/fpsyg.2011.00266

Higgins, E. T. (1987). Self-discrepancy: A theory relating self and affect. Psychological Review, 94(3), 319-340. https://doi.org/10.1037/0033-295X.94.3.319

Holmes, E. A., \& Mathews, A. (2010). Mental imagery in emotion and emotional disorders. Clinical Psychology Review, 30(3), 349-362. https://doi.org/10.1016/j.cpr.2010.01.001 
IBM Corp. Released 2020. IBM SPSS Statistics for Mac, Version 28.0. Armonk, NY: IBM Corp

Kilborne, B. (1997). Self-Conscious Emotions: The Psychology of Shame, Guilt, Embarrassment, and Pride. The Journal of Nervous \&amp Mental Disease, 185(6), 413. https://doi.org/10.1097/00005053-199706000-00012

Kuznetsova, A., Brockhoff, P.B., Christensen, R.H.B., and Jensen, S.P. (2020). lmerTest. Leary, M. R. (2007). Motivational and Emotional Aspects of the Self. Annual Review of Psychology, 58(1), 317-344.

https://doi.org/10.1146/annurev.psych.58.110405.085658

Lenth, R. (2019). emmeans: estimated marginal means, aka least-squares means.

Libby, L. K., \& Eibach, R. P. (2002). Looking back in time: Self-concept change affects visual perspective in autobiographical memory. Journal of Personality and Social Psychology, 82(2), 167-179. https://doi.org/10.1037/0022-3514.82.2.167

Libby, L. K., \& Eibach, R. P. (2011). Visual perspective in mental imagery: A representational tool that functions in judgment, emotion, and self-insight. In J. M. Olson \& M. P. Zanna (Eds.), Advances in experimental social psychology, (Vol. 44, pp. 185-245). Academic Press. https://doi.org/10.1016/B978-0-12-385522-0.00004-4

Libby, L. K., Eibach, R. P., \& Gilovich, T. (2005). Here's looking at me: The effect of memory perspective on assessments of personal change. Journal of Personality and Social Psychology, 88(1), 50-62. https://doi.org/10.1037/0022-3514.88.1.50

Lüdecke D (2021). sjPlot: Data Visualization for Statistics in Social Science. R package. McAdams, D. P. (1996). Personality, Modernity, and the storied self: A contemporary framework for studying persons. Psychological Inquiry, 7(4), 295321. https://doi.org/10.1207/s15327965pli0704_1 
McAdams, D. P., \& Pals, J. L. (2006). A new Big Five: Fundamental principles for an integrative science of personality. American Psychologist, 61(3), 204217. https://doi.org/10.1037/0003-066X.61.3.204

Mcisaac, H. K., \& Eich, E. (2002). Vantage point in episodic memory. Psychonomic Bulletin and Review, 9(1), 146-150. https://doi.org/10.3758/BF03196271

Metzinger, T. (2007). Empirical perspectives from the self-model theory of subjectivity: a brief summary with examples. Progress in Brain Research, 168(07), 215-245. https://doi.org/10.1016/S0079-6123(07)68018-2

Miceli, M., \& Castelfranchi, C. (2018). Reconsidering the Differences Between Shame and Guilt. Europe's Journal of Psychology, 14(3), 710-733. https://doi.org/10.5964/ejop.v14i3.1564

Mills, R.S.L., Hastings, P.D., Serbin, L.A. et al. Depressogenic Thinking and Shame Proneness in the Development of Internalizing Problems. Child Psychiatry Hum Dev 46, 194-208 (2015). https://doi.org/10.1007/s10578-013-0416-4

Nigro, G., \& Neisser, U. (1983). Point of view in personal memories. Cognitive Psychology, 15(4), 467-482. https://doi.org/10.1016/0010-0285(83)90016-6

Robinson, J. A., \& Swanson, K. L. (1993). Field and Observer Modes of Remembering. Memory, 1(3), 169-184. https://doi.org/10.1080/09658219308258230

RStudio Team (2019). RStudio: Integrated Development for R. RStudio, Inc., Boston, MA URL http://www.rstudio.com/

Sanitioso, R. B. (2008). Motivated self and recall: visual perspectives in remembering past behaviors. European Journal of Social Psychology, 38(3), 566575. https://doi.org/10.1002/ejsp.456 
Schalkwijk, F., Stams, G.J., Dekker, J.J., Peen, J., \& Elison, J. (2016). Measuring Shame Regulation: Validation of the Compass of Shame Scale. Social Behavior and Personality, 44, 1775-1792.

Schoenleber, M., \& Berenbaum, H. (2012). Shame regulation in personality pathology. Journal of Abnormal Psychology, 121(2), 433446. https://doi.org/10.1037/a0025281

Singer, J. A., \& Blagov, P. (2004). The Integrative Function of Narrative Processing: Autobiographical Memory, Self-Defining Memories, and the Life Story of Identity. In D. R. Beike, J. M. Lampinen, \& D. A. Behrend (Eds.), The self and memory (pp. 117138). Psychology Press.

St Jacques, P. L., Carpenter, A. C., Szpunar, K. K., \& Schacter, D. L. (2018). Remembering and imagining alternative versions of the personal past. Neuropsychologia, 110, 170179. https://doi.org/10.1016/j.neuropsychologia.2017.06.015

St Jacques, P. L., Szpunar, K. K., \& Schacter, D. L. (2017). Shifting visual perspective during retrieval shapes autobiographical memories. NeuroImage, 148, 103-114. https://doi.org/10.1016/j.neuroimage.2016.12.028

Sutin, A. R., \& Robins, R. W. (2008). When the "I" looks at the "Me": Autobiographical memory, visual perspective, and the self. Consciousness and Cognition, 17(4), 13861397. https://doi.org/10.1016/j.concog.2008.09.001

Tangney, J. P., Stuewig, J., \& Mashek, D. J. (2007). Moral Emotions and Moral Behavior. Annual Review of Psychology, 58(1), 345-372. https://doi.org/10.1146/annurev.psych.56.091103.070145

Tangney, June P. (2015). Selfconscious Emotions, Psychology of. International Encyclopedia of the Social \& Behavioral Sciences: Second Edition (2nd ed., pp. 475-480). Elsevier. https://doi.org/10.1016/B978-0-08-097086-8.25032-0 
Tangney, June Price, Wagner, P. E., Hill-Barlow, D., Marschall, D. E., \& Gramzow, R. (1996). Relation of Shame and Guilt to Constructive Versus Destructive Responses to Anger Across the Lifespan. Journal of Personality and Social Psychology, 70(4), 797809. https://doi.org/10.1037/0022-3514.70.4.797

Tangney, June Price, Wagner, P., \& Gramzow, R. (1992). Proneness to Shame, Proneness to Guilt, and Psychopathology. Journal of Abnormal Psychology, 101(3), 469-478. https://doi.org/10.1037/0021-843X.101.3.469

Teroni, F., \& Deonna, J. A. (2008). Differentiating shame from guilt. Consciousness and Cognition, 17(3), 725-740. https://doi.org/10.1016/j.concog.2008.02.002

Thompson, E. R. (2007). Development and validation of an internationally reliable shortform of the positive and negative affect schedule (PANAS). Journal of cross-cultural psychology, 38(2), 227-242. https://doi.org/10.1177/0022022106297301

Welten, S. C. M., Zeelenberg, M., \& Breugelmans, S. M. (2012). Vicarious shame. Cognition and Emotion, 26(5), 836-846. https://doi.org/10.1080/02699931.2011.625400

Wilson, A. E., Ross, M., \& Wilson, A. E. (2010). The identity function of autobiographical memory : Time is on our side The identity function of autobiographical memory : Time is on our side. Psychology, 11, 37-41.

Zaragoza Scherman, A., Salgado, S. Shao, Z., Berntsen, D. (2015) Event centrality of positive and negative autobiographical memories to identity and life story across cultures, Memory, 23(8), 1152-1171, DOI: 10.1080/09658211.2014.962997 